\title{
THE SUPPLY AND UTILIZATION OF DENTAL SERVICES
}

\author{
P. P. Groenewegen and J. H. M. Postma
}

Netherlands Institute of General Practitioners, P.O. Box 812, 3500 AV Utrecht, The Netherlands

\begin{abstract}
In this article the question is addressed whether regional differences in the supply of dental manpower influences the utilization of dental services. The percentage of the population that visits the dentist, is indeed higher in regions with a higher density of dentists. The number of people that visit a dentist is higher among privately insured patients than among the publicly insured. We have constructed a simple model of the behavior of dentists and patients to find out whether this difference is influenced by the supply of dental manpower. On the assumption that dentists prefer the treatment of privately insured patients we predict a greater difference between publicly and privately insured patients in regions with a lower density of dentists than in regions with a higher density. The data does not unequivocally support this prediction. A second assumption is that differences between social groups in the chances of uptake of regular treatment influence the behavior of patients on future points in time. To find out whether the limited data we have, support this assumption, we have looked at the differences between privately and publicly insured patients in regions that show an important change in density. The results of this analysis are not as predicted.
\end{abstract}

\section{INTRODUCTION}

The two subjects in the title of this report are in general treated separately. The geographical distribution of dentists has been documented, e.g. the United States [1], United Kingdom [2] and The Netherlands [3]. The utilization of dental services has been studied more frequently [4]. Research in the use of dental services is in general directed to finding factors that influence the frequency of visits to the dentist; such as age and socio-economic status. These factors can be interpreted as indicators of the demand for services. For a more comprehensive study of utilization, demand as well as supply have to be taken into account [5]. O'Mullane and Robinson [6] have done this by comparing the uptake of dental treatment in two towns with widely different dentist to population ratios. Their conclusion is that there is a difference in the number of visits to a dentist between children of higher and lower social strata and this difference is greater in the town with the lower dentist to population ratio than in the town with the higher ratio. The first question we put forward in this paper is whether the frequency of visits to a dentist in The Netherlands is influenced by regional differences in the density of dentists. The second question is whether there are theoretical reasons to expect a differential effect for some sections of the population and if so, whether this can be demonstrated in the data at hand.

A description of the organization of dental health care in The Netherlands is given with a survey of some indicators of the demand for dental care. Outlines of an explanation of the influence of demand and supply on the utilization of dental services are sketched. Central to this explanation is a simple model of the behaviour of patients and dentists. The behaviour of dentists and patients is combined in a dependency model in the sense in which Boudon [7] uses this term. We will then show some of the results of our analysis of the supposed influence of regional differences in the density of dentists on the utilization of dental services for different social groups.

\section{THE ORGANIZATION OF DENTAL HEALTH CARE IN THE NETHERLANDS}

The Dutch health care system consists of an interesting mix of private initiative and public regulation. Most of the individual providers of health services are private entrepreneurs and even institutions like hospitals are mostly owned and run by private foundations [8]. The transactions between providers and patients are regulated by the law, but the enactment of the law is in the hands of private institutions-the sickfunds. The result is a rather intricate system of control and deliberation. The Sickfunds Act (1964) regulates most of the transactions in the health care sector [9]. Employees with a yearly income below Hfl. 43,500.00 (in 1982) are legally insured for the costs of medical care. Self-employed and elderly people with a low income can choose for sickfund insurance. Private insurance companies cover the rest of the population. There are as a rule no direct financial transactions between providers and publicly insured patients [10]. Employees and employers each pay half of the premium to the sickfund; the providers are payed by the sickfund on a fee for service basis (as is the case for dentists and medical specialists) or as a capitation fee (general practitioners). Privately insured patients pay the providers for their services and are as far as the coverage of their insurance goes, restituted by the private insurance companies.

Dental health care traditionally has a peripheral position in the health care system. The provision of dental care is an isolated activity, in part as a result of the organization of practice-the majority of dentists are in single-handed practices-and in part as a result of the clearcut task of dentists compared with that of other health care professionals. With a few exceptions (e.g. a number of dentists who work in a special clinic for publicly insured patients) den- 
Table 1. Percentage of the respondents that visited a dentist in the year that preceded the survey, by age-group

\begin{tabular}{|c|c|c|c|c|c|c|}
\hline & \multicolumn{6}{|c|}{ Age } \\
\hline & $\leqslant 20$ & $21-30$ & $31-40$ & $41-50$ & $51-60$ & $>60$ \\
\hline $\begin{array}{c}\% \\
\text { Number }\end{array}$ & $\begin{array}{l}85.2 \\
351\end{array}$ & $\begin{array}{l}77.1 \\
887\end{array}$ & $\begin{array}{l}67.5 \\
871\end{array}$ & $\begin{array}{l}48.2 \\
633\end{array}$ & $\begin{array}{l}33.1 \\
589\end{array}$ & $\begin{array}{l}16.7 \\
826\end{array}$ \\
\hline
\end{tabular}

tists are in private practice. Dental care is directly accessible and therefore belongs to the first line of health care together with, for example, general practitioners and public health nurses. Dental care for children in primary school is organized in school dental services. These services cover approximately half of the children in primary school age.

The publicly insured part of the population is entitled to a limited number of services, the so called dental care according to systematic rational principles. These services are the treatment of complaints of pain, the extraction of elements that will probably not be maintained, scaling of the teeth and instruction in dental hygiene, simple conserving treatment and prosthetic help to restore the chewing function [11]. These services are completely free for publicly insured patients who visit the dentist regularly (twice a year). Those who do not visit the dentist regularly or who want other services than those listed above have to pay (part of) the fee themselves. The greater part of the privately insured patients are not covered for dental care costs [12]. A number of companies, however, cover the costs of dental care for children.

\section{THE DEMAND FOR DENTAL SERVICES}

When one studies the influence of regional differences in the supply of dental services on utilization, one has to take into account some indicators of the demand for services, most notably the age of the potential patient. The number of people with full dentures who will therefore hardly visit a dentist anymore, rises with age. Other important indicators are socio-economic position, level of education and the insurance for the costs of dental care.

\section{Age}

The influence of age on the demand for dental care is mediated by the number of people with full dentures. Unfortunately there were no questions asked on this subject in the survey that we used in our analysis [13]. In a recent national health survey [14] respondents were asked whether they still have their own teeth. In the group of respondents aged 16-29 years only $2.6 \%$ has full dentures. For those aged $30-39$ years $14.7 \%$; 40-49 years $32.1 \%$; $50-59$ years $56 \%$ and for respondents aged 60 years and older $75.4 \%$. The percentage of the population that visited a dentist at least once a year preceding the survey, therefore varies with age. To exclude some of the influence of age and-indirectly-of the number of people with full dentures, we have conducted the analysis in this article for people aged 40 years and under.

\section{Socio-economic status}

Research in different health care systems reveals that people with a higher socio-economic status visit the dentist more frequently than people of lower status [15]. As indicators of socio-economic status occupation or a combination of occupation. education and income are most frequently used. In Table 2 the percentage of the population aged 40 years and under that visited a dentist at least once in the year preceding the survey, is given for the cross-tabulation of income and education [16]. Level of education and income both have an influence on the percentage of people that visit a dentist. The relationship is, however, not completely additive. The percentage in the high education/high income cell is lower than would be expected under the assumption of additivity, whereas the percentage in the low education/high income cell is higher.

\section{Insurance for the costs of dental care}

The final influence on the frequency of visits to a dentist is the insurance for the costs of dental care. The frequency of visits is lower for the publicly insured part of the population. In an analysis of the same data Mootz [17] found that $46 \%$ of the publicly insured payed at least one visit to a dentist in the year preceding the survey against $64 \%$ of the privately insured for all age-groups. This difference is in part the result of differences in socio-economic status, in level of education and in the number of people with full dentures. Because of the income limit in the public insurance scheme the publicly insured are in general from lower socio-economic strata than the privately insured. The number of people with full dentures is also greater under the publicly insured. The National Health Survey [14] shows that $35 \%$ of all the publicly insured have full dentures against $24^{\circ} \%$ of the privately insured. In the 40 years and under age group $72 \%$ of the publicly insured and $81 \%$ of the privately insured visited a dentist in the year preceding the survey. When this difference is broken down by level of education and by income it appears that the difference is only found in the low education and low income groups (although the number of respondents in the low income/privately insured group is of course very small).

\section{THE SUPPLY OF DENTAL SERVICES}

The volume of dental care in a region depends on two factors: (i) the number of active dentists in the region in relation to the population and (ii) the productivity of dentists. Productivity varies with the

Table 2. Percentage of the respondents ( $\leqslant \mathbf{4 0}$ years) that visited a dentist in the year preceding the survey, by income and level of education

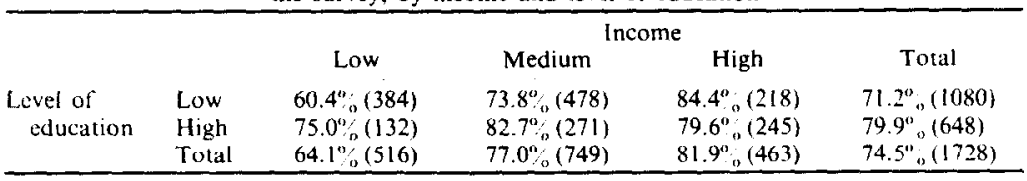


Table 3. Percentage of the respondents ( $\leqslant 40$ years) that visited a dentist in the year preceding the survey, by type of insurance and level of education

\begin{tabular}{|c|c|c|c|}
\hline & & \multicolumn{2}{|c|}{ Type of insurance } \\
\hline & & Public & Private \\
\hline $\begin{array}{l}\text { Level of } \\
\text { education }\end{array}$ & $\begin{array}{l}\text { Low } \\
\text { High } \\
\text { Total }\end{array}$ & $\begin{array}{l}68.5 \%(1084) \\
80.9^{\circ} \%(440) \\
72.1 \%(1524)\end{array}$ & $\begin{array}{l}81.7 \%(246) \\
80.7 \%(290) \\
81.2 \%(536)\end{array}$ \\
\hline
\end{tabular}

Table 4. Percentage of the respondents ( $\leqslant 40$ years) that visited a dentist in the year preceding the survey, by type of insurance and income

\begin{tabular}{|c|c|c|c|}
\hline & & \multicolumn{2}{|c|}{ Type of insurance } \\
\hline & & Public & Private \\
\hline Income & $\begin{array}{l}\text { Low } \\
\text { Medium } \\
\text { High }\end{array}$ & $\begin{array}{l}62.7 \%(469) \\
76.7^{\circ} \%(574) \\
80.4^{\circ} \%(235)\end{array}$ & $\begin{array}{l}82.2 \%(45) \\
78.3 \%(175) \\
83.6 \%(232)\end{array}$ \\
\hline
\end{tabular}

amount of delegation to auxiliaries and with experience or dexterity of the dentist. Dexterity seems to be at its top around 45 years [18]. However, the data on the geographical distribution of dentists that we use for our analysis do not contain the age distribution of the dentists. The geographical distribution of dentist was coded from the 'Tandartsengids' (annual list of dentists, edited by the Dental Association in The Netherlands), which contains the name and address of all dental practices. As an indicator of the regional differences in the supply of dental services we used the density of dentists, defined as the number of inhabitants of a region divided by the number of dentists in that region. This is probably not the best indicator of the supply of dental services, while it does not take into account differences in the productivity of dentists. Furthermore there is the problem of patients living in one region and visiting a dentist who practices in another region. It is, however, the best indicator at hand.

The first question we asked in the introduction of this article is whether the supply of dental services influences the utilization. Table 5 gives the relevant figures. Table 5 indicates that there is indeed an influence of the supply of dental services on utilization. The percentage of people that visited a dentist in the year preceding the survey is greater for people that live in areas with a higher density of dentists. However, the differences are rather small. The second question we put forward is whether theoretically a differential effect for different social groups can be expected. A common explanation of differences in the number of visits to a dentist is in terms of the value and importance people attach to maintaining one's own teeth and to dental hygiene in general. Such an explanation is not exhaustive. It is difficult to imaginc why people in areas with a higher density of dentists would attach more importance and value to their teeth than people in areas with a lower density of dentists. Moreover, from this common explanation one cannot predict a greater difference in the frequency of visits to a dentist between people of higher and lower strata in areas with a lower density of dentists than in areas with a higher density (as was found by O'Mullane and Robinson). As Frazier [5] points out: "... explanations for the under-utilization of dental services by low socio-economic families, including those for whom financial barriers have been removed, perhaps may rest less upon social class differences in the value placed on dental care, than upon structural and interpersonal factors which affect the acceptability of the services".

\section{DIFFERENCES IN THE UTILIZATION OF DENTAL SERVICES BETWEEN SOCIAL GROUPS UNDER THE CONDITION OF DIFFERENT LEVELS OF SUPPLY}

A more complete explanation has to take structural factors into account. This can be done by constructing a simple model of the dependency system, formed by the behaviour of patients of different social groups and the behaviour of dentists, under the condition of differences in the density of dentists and given the peculiarities of the dental health care system in The Netherlands. The model can be outlined as follows. We distinguish three groups of actors: the patients who are publicly insured, the patients who are privately insured (this amounts in part to the difference between people from lower and people from higher social strata) and the dentists in general practice. The behavioural alternatives concerned are for the patients to visit a dentist or not. For the dentists the behavioural alternatives are the treatment of publicly or privately insured patients under the assumption of maximization of income, free time and work satisfaction. Given the organization of the (dental) health care system in The Netherlands it is the dentists, under the condition of a low density of dentists, that find it more profitable to treat privately insured patients than publicly insured patients. Dentists are free to decide how many publicly insured patients they enter on their list. Publicly insured patients have a right to certain services; the sickfund is obliged to make-as far as possibie-the services available, but the providers of care have the right to refuse patients in order to retain a reasonable list-size. Under the condition of low density of dentists, this results in a higher probability of regular treatment for privately insured patients-even when the behavioural parameters of the publicly and privately insured patients are equal. This outcome could have a feedback effect on the behavioural parameters of patients for future points in time. Theoretically, the lower probability of treatment of publicly insured patients could result in a lower subjective probability of keeping one's own teeth as a result of visiting a dentist. This, as shall be outlined more fully, results

Table 5. Percentage of the respondents ( $\leqslant 40$ years) that visited a dentist in the year preceding the survey. by density of dentists

\begin{tabular}{lcccc}
\hline \multicolumn{4}{c}{ year preceding the survey. by density of dentists } \\
\hline & $\begin{array}{c}\text { Number of inhabitants per dentist } \\
\leqslant 3000\end{array}$ & & & \\
(High density) & $3001-4000$ & $4001-5000$ & (Low density) \\
\hline Number & 78.0 & 76.7 & 74.9 & 69.8 \\
\hline & 554 & 420 & 430 & 656 \\
\hline
\end{tabular}


in a lower subjective expected utility of a visit to a dentist for publicly insured patients.

\section{The behaviour of dentists and patients}

Rational choice theory provides a model of the behaviour of the actors [19]. The core of rational choice theory is that behavioural alternatives have different consequences, that people value these consequences positively or negatively, that the valuations can be rank-ordered and that the consequences of behavioural alternatives do not occur with certainty, but that people estimate the probability with which they expect the consequences to occur. As far as the patients are concerned we are interested in the behavioural alternatives of going to the dentist or not. A positive consequence of going to the dentist is-as we assume-maintaining one's own teeth, while the negative consequences are the cost of treatment and the cost of foregone opportunities in the time it takes to travel to the dentist, the waiting time and the duration of treatment. To keep the model simple we neglect other possible consequences of the choice to go to the dentist or not (e.g. pain or anxiety [20]). We also assume that the estimated probability of incurring costs is one, while the estimated probability of maintaining one's own teeth as a consequence of visiting the dentist has a value somewhere between 0 and 1. Differences between publicly and privately insured patients in the frequency of visits to the dentist can now be traced to these parameters. Differences can be explained by differences in the value attached to maintaining one's own teeth and/or the estimated probability of maintaining one's own teeth as a consequence of visiting the dentist and/or in the costs.

The fact that in The Netherlands people with higher income, people with a higher level of education and people who are privately insured visit the dentist more frequently can perhaps in part be explained by differences in the value people attach to their teeth. The difference cannot be explained by higher costs of a visit to the dentist for people of lower strata. In general the costs will be higher for people of the higher social strata who are privately insured and therefore have to pay the fee of the dentist themselves [21]. A relation such as the one found by O'Mullane-greater differences between people of higher strata and people of lower strata in areas with a lower density of dentists-also cannot be attributed to differences in values and costs. As we have said it is generally found in empirical research that people of higher strata place more value in maintaining their own teeth, but it is difficult to imagine why there should be greater differences between people of higher and lower strata in this value in areas with a lower density of dentists. The effect of social background and the density of dentists must therefore at least in part be attributed to differences in the estimated probabilities and the behaviour of dentists.

In our model of the behaviour of dentists we assume that the behavioural alternatives are the treatment of privately insured patients or the treatment of publicly insured patients. The consequences of the choice of an alternative are in the realm of the amount of income that dentists gain out of their practice, the number of hours of free time and the amount of work satisfaction. To simplify the matter we assume that individual differences between dentists in the value they attach to income, free time and work satisfaction and in the estimations of probabilities are not systematic and are levelled out in the aggregate. The amount of income that can be gained from dental practice is higher-given the Dutch system of dental care-when a dentist treats more privately insured patients. Due to the fact that the provision of dental care to publicly insured patients is limited to dental care according to systematic rational principles the work satisfaction from the treatment of publicly insured patients is also assumed to be lower than the treatment of privately insured patients. The relation between income and free time is assumed to be a simple one: free time is total time minus the time for treating publicly insured patients and the time for treating privately insured patients. It can be assumed that treating publicly insured patients is less time-consuming than the treatment of private patients. So in principle dentists could optimize the ratio of income and free time by treating more publicly insured patients. However, the level of the fees is based among other things on the duration of treatment. Now we can analyse the dependency system of the behaviour of dentists and patients given the organizational environment of the Dutch dental health care system.

\section{The dependency model}

As far as the behaviour of dentists is concerned, we assume that the nett utility of treating privately insured patients is higher than that of treating publicly insured patients. Under the structural condition that there is a low density of dentists, all private patients that seek treatment will be treated and publicly insured patients will be treated until the ratio of income and free time reaches its optimum. As far as the differences in utility of going to the dentist or not for privately and publicly insured patients are concerned, we assume that the nett utility of going to the dentist is greater or the same for private patients than for publicly insured patients. For the first group the costs are higher but they value their teeth more; for the latter group costs are lower but in general it is assumed that they attach less value to their teeth. The outcome of this interaction system is that the probability of regular treatment by a dentist is greater for privately insured patients than for publicly insured, given the structural condition of low density of dentists, even when there would be no utility differences between private and publicly insured patients. Our prediction, therefore, for an analysis at one point in time is that the difference in frequency of visits to a dentist between the privately and publicly insured patients is greater for people that live in an area with a low density of dentists than it is for people in an area with a high density. This prediction cannot be transposed to a dynamical situation without further analysis of the interaction system. The number of dentists increases rapidly in The Netherlands. The areas with a high density of dentists at a certain point in time can be divided in areas with also a high density of dentists at an earlier point in time (no major change in density) and areas in which there has been an increase in density. The question is: what 


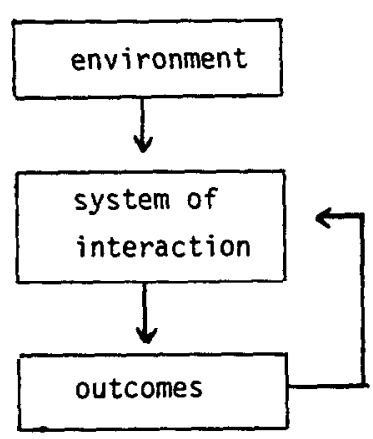

Fig. 1. General scheme of a feedback system (Source: Boudon [7]).

happens to the difference between publicly and privately insured patients in the frequency of visits to a dentist when the density of dentists increases. Our hypothesis is that there is a feedback relation from the outcome of the interaction system of the behaviour of dentists and patients under different conditions of density of dentists to the interaction system at a following point in time. More specifically this feedback is to the estimated probability of maintaining one's own teeth as a consequence of visiting the dentist. The general scheme of these feedback systems is given by Boudon [7, 22] (Fig. 1).

The outcome of the interaction system is that publicly insured patients have-under the condition of a low density of dentists-a smaller probability of regular treatment. This outcome however, influences the utility differences at a following point in time. In general the estimated probabilities of the occurrence of consequences of actions are influenced by what happens in the past in identical or comparable situations to oneself or to significant others. When people do not take up regular dental treatment and/or people in their direct social environment do not take up regular treatment, the estimated probability of maintaining one's own teeth as a consequence of visiting the dentist is lowering down (and the probability of a more painful treatment rises). The outcome of this process is that in areas with a low density of dentists at $t_{1}$, the difference in nett utility of going to the dentist for privately and publicly insured patients is becoming greater. As a consequence the difference in the frequency of uptake of dental treatment is greater at $t_{2}$. Further, it could be predicted that this effect does not completely disappear when the structural condition of density of dentists changes. The areas with a relatively high density at $t_{2}$ can be divided in those areas with a low density at $t_{1}$ and those with a high density at $t_{1}$.

We expect to find a greater difference in the frequency of visits to the dentist between publicly and privately insured patients in areas with a lower density at $t_{1}$ and at $t_{2}$, but also in areas with a lower density at $t_{1}$ and a higher density at $t_{2}$ (Fig. 2).

\section{A PROVISIONAL TEST OF} THE PREDICTIONS

The first part of the second question we put forward in the introduction-whether there are the- oretical reasons to expect a differential effect of regional differences in the density of dentists for some sections of the population-has been answered in the positive. We now come to the second part of the question-whether this can be demonstrated in the data at hand. Because of the nature of the data we use, this can only be termed a provisional test of the predictions. The underlying assumptions cannot be tested. In Table 6 the percentage of the respondents that visited a dentist is broken down by the density of dentists, type of insurance and level of education. In all categories of the density of dentists there is a difference between publicly and privately insured patients (although the difference is very small in the category with $3001-4000$ inhabitants per dentist). The difference between the percentages for the publicly and privately insured patients are respectively 10,2 , 16 and $8 \%$. Although there is a slight tendency for the differences to be greater when the density of dentists is lower, this is not true for the two extreme categories; only the two intermediate categories show the predicted difference. It is already shown in Table 3 that there are virtually no differences between publicly and privately insured patients with a high level of education. In three out of four categories in Table 6 publicly insured patients with a high education have an even higher percentage than the privately insured patients with a higher level of education. Within the category of people with a low level of education we expect the differences between publicly and privately insured patients to be greater for people in areas with a lower density of dentists. The differences between the percentages for the publicly and privately insured patients with a low level of education are respectively $16,2,22$ and $13 \%$. In this case also the expected difference is only found in the two intermediate categories, but not in the extreme categories.

That we do not find the predicted effects in the extreme categories suggests that our predictions are nol accurate or incomplete. Another possibility is that the category of people living in areas with a high density of dentists (less than 3000 inhabitants per dentist) should be divided in people in areas that experienced only a minor change in density in the past years and those in areas that experienced an increase in density. In areas that for a longer period had a high density of dentists, we expect to find only small difference between publicly and privately insured patients, while in areas that had an increase in density, we expect a greater difference between publicly and privately insured patients, according to the feedback mechanism suggested above. In Table 7 the people living in areas with a high density of dentists (less than 3000 inhabitants per dentist) are subdivided into three categories: those with no change or a minor change in density between 1969 and 1977 [23] (i.e. the difference in the number of inhabitants per dentist in 1969 and 1977 is less than 550), those with a higher density in 1977 than in 1969 (difference in the number of inhabitants per dentist between 550 and 1200) and those areas with a much higher density in 1977 than in 1969 (a difference of more than 1200 inhabitants per dentist). The differences between publicly and privately insured patients in the percentage that visited a dentist at least once in the year preceding the survey, are respectively 16,3 and $22 \%$. The difference 
$t_{1}$

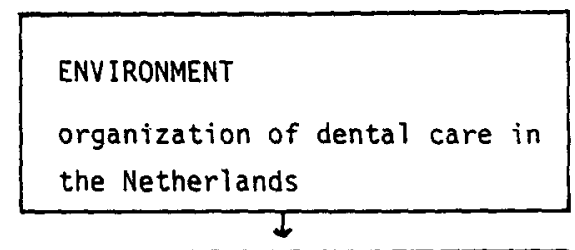

INTERACTIONSSYSTEM

actors: publicly insured

patients, privately insured

patients, dentists

behavioral alternatives of the

patients: visiting a dentist

or not

behavioral alternatives of the

dentists: treatment of publicly

insured patients or of

privately insured patients

differences in nett utility

between alternatives: nett

utility of visiting a dentist

is for privately insured

patients higher or the same as

for publicly insured patients;

nett utility for dentists of

treating privately insured

patients is greater than of

treating publicly insured

patients.

structural condition: low

density of dentists

I

OUTCOME
probability of regular treat-
ment is greater for privately
insured patients

$t_{2}$

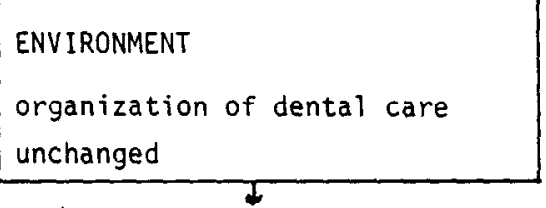

INTERACTIONSSYSTEM

structural condition: density

of dentists at $t_{2}$ is the same

or higher than at $t_{1}$

differences in nett utility

between alternatives: as a

result of the low probability

of regular treatment of

publicly insured patients the

subjective estimated

probability of maintaining

one's teeth through visiting

a dentist goes down;

irrespective of the difference

in nett utility between

privately and publicly insured

patients at $t_{1}$, therefore, the

nett utility difference between

both categories of patients

becomes greater

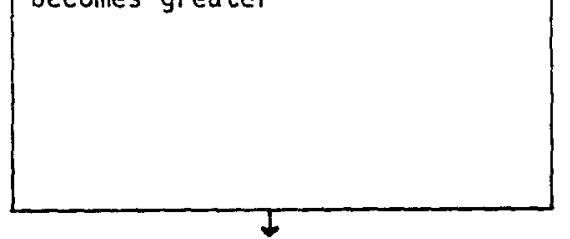

OUTCOME
greater difference in frequency
of visits to a dentist between
publicly and privately insured
patients

Fig. 2. Outline of a cumulative feedback system to explain the difference in visits to a dentist between publicly and privately insured patients.

between publicly and privately insured patients in areas with no change or only a minor change in density, is much greater than expected. Moreover, when we confine ourselves to the people with a relatively low level of education, the results are inconsistent with the prediction.

\section{DISCUSSION}

In the preceding sections we have showed that the supply of dentists - as measured by the number of inhabitants per dentist-has some influence on the utilization of dental services. From an analysis of the behavioural alternatives of publicly and privately insured patients and of dentists we predicted a greater difference in the percentage that visit a dentist, between publicly and privately insured patients in areas with a low density of dentists than in areas with a higher density. An analysis of the data not unequivocally supports this prediction. In the intermediate categories of the density of dentists the results are as predicted, but in the extreme categories, 

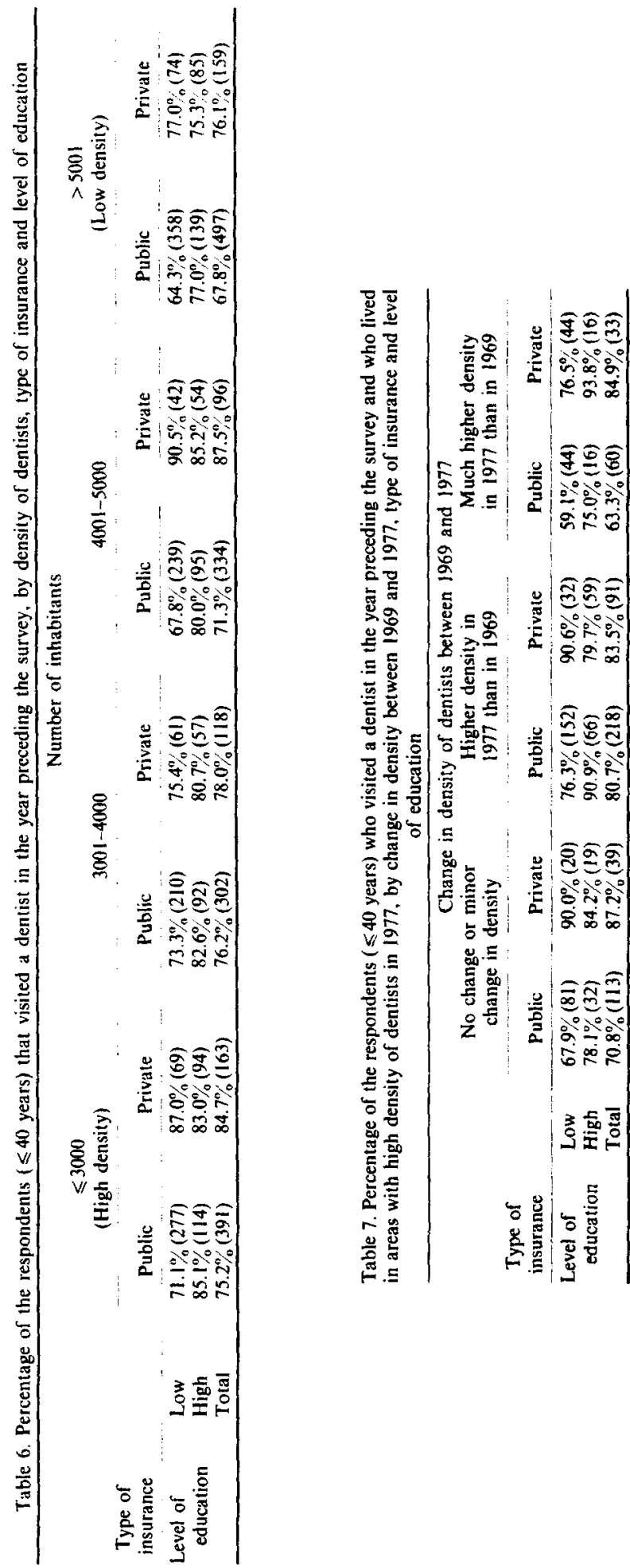
which are more important in the test of the prediction, the results are inconsistent with the prediction. The model with which we made this prediction, was built on the assumption that there are no great differences in nett utility of visiting a dentist between publicly and privately insured patients and that dentists prefer the treatment of privately insured patients. When there are only relatively few dentists in a region, they use most of their working time treating private patients. In areas with a higher density they have to take up treatment of publicly insured patients to assure a reasonable income. The outcome is, according to the model, that publicly insured patients in areas with a low density of dentists have a smaller change of regular treatment. Our second hypothesis was that this influences their behaviour at a future point in time, more specifically through a lower subjective estimation of the probability of maintaining one's own teeth through visits to the dentist. We formulated this hypothesis to explain an observation that one could find in the Dutch papers a few years ago, when the community of Rotterdam installed some dentists in community owned and exploited premises to reduce the shortage of dentists. These new dentists, however, had not enough work, because people did not use their services. The common explanation was that people had lost the habit of regular visits to a dentist.

The fact that our predictions did not work out, can be attributed to the model itself or to the data we had to test the implications of the model. Perhaps there are too many untested steps in our model to find easily interpretable results, in particular in our assumptions about the behavioural parameters of different groups of patients. When it would be possible to distinguish between regular, preventive visits to a dentist and visits because of complaints and when there are data on visits to a dentist over a longer period in a person's life, probably more specific predictions could be tested. Also our indicator of the supply of dental services could be improved. All in all we think that research into the utilization of dental services could benefit from the distinction between valuations and estimations of the probability of maintaining one's own teeth and from the explicit consideration of the structural conditions of supply.

Acknowledgements - We like to thank the Centraal Bureau voor de Statistiek for the permission to use the data for this analysis and F. L. Leeuw, R. Wippler and our colleagues in the research department of The Netherlands Institute of General Practitioners for their comments on an earlier draft of this paper.

\section{REFERENCES}

1. American Dental Association, Bureau of Economic and Behavioral Research. Distribution of dentists in the United States by state, region, district and county, Chicago, 1979, American Dental Association, Bureau of Economic and Behavioral Research, Manpower Resource Book, Chicago, 1980.

2. Cook P. J. and Walker R. O. The geographical distribution of dental care in the United Kingdom. Part 1: sources of information; methods of presentation; distribution of dentists-part 2: changes between 1952 and 1962-part 3: regional synthesis and discussion. $B r$. dent. J. 16 May, 1967; G June, 1967; 20 June, 1967.
3. Groenwegen P. P. and van der Zee J. De spreiding van huisartsen over Nederland, deel 2: huisartsen en tandartsen vergeleken (A comparison of the geographical distribution of general practitioners and dentists) Netherlands Institute of General Practitioners. Utrecht. 1982. Groenewegen P. P. and Postma J. H. M. De geografische spreiding van tandartsen over Nederland (The geographical distribution of dentists in the Netherlands). Nederlands Tijdschrift voor Tandheelkunde. In press.

4. A survey of the literature is given by Richards N. D. Utilization of dental services. In Social Sciences and Dentistry (Edited by Richards N. D. and Cohen L. K.). Den Haag, 1971.

5. As has been emphasized by Frazier P. J., Jenny J.. Bagramian R. A., Robinson E. and Proshek J. M. Provider expectations and consumer perceptions of the importance and value of dental care. Am. J. publ. Hith 67, 37-43, 1977 .

6. O'Mullane D. M. and Robinson M. E. The distribution of dentists and the uptake of dental treatment by schoolchildren in England. Communit. Dent. Oral Epid. 5, 156-159, 1977.

7. Boudon R. La Logique du Social. Hachette, Paris, 1979.

8. Rutten F. and van der Werff A. Health policy in The Netherlands at the crossroads. In The Public/Private Mix for Health (Edited by McLachlan G. and Maynard A.). The Nuffield Provincial Hospital Trust, London. 1982.

9. Another important law is the General Act for Special Sickness Expenses (1967) which covers among other things long term stay in hospitals and nursing homes.

10. Of course there are a few exceptions; not everything is included in the insured risk (e.g. abortion) and since February 1983 publicly insured have to pay a small amount for each pharmaceutical prescription.

11. Decree Provision of Dental Care to Publicly Insured; these limitations do not apply to children aged 13 years from 1974 on who are treated under the Decree Integral Dental Care for Publicly Insured.

12. With the exception of civil servants who have an insurance scheme that also covers $80 \%$ of the costs of dental care.

13. We thank the Centraal Bureau voor de Statistiek (Central Bureau of Statistics) for the permission to use the data of the 'Leefsituatie-onderzoek 1977' (Life Situation Survey).

14. van den Berg J. Gebitsprothesen bij de Nederlandse Bevolking (Dental Prostheses among the Dutch population). Maandber. Gezond. October, 1982.

15. Butler J. R. Studies in the use of dental services. Soc. Econ. Admin. 1, 5-18, 1967. Davis P. Culture, inequality and the pattern of dental care in New Zealand. Soc. Sci. Med. 15A, 801-805, 1981. Van Groenestijn M. A. J., Maas-de Waal C. J., Swallow J. N. and Mileman P. A. Regelmatig-niet regelmatig tandartsbezoek (Visit to the dentist: regular and non-regular attendance). Tijdsch. Soc. Geneesk. 57, 369-375, 1979.

16. The variable 'level of education' (var. 0022 ØP) has originally four categories, but has been recoded into two for this analysis. The variable 'income' has seven categories and has been recoded into three categories. For $18 \%$ of the respondents of 40 years or younger the nett income of the respondent was coded as 'missing'.

17. Mootz M. Ziekenfondslidmaatschap en het bezoek aan de tandarts (Type of insurance and visits to the dentist) Nederlands Tijdsch. Tandheelk. 86, 76-79, 1979.

18. Gray A. M. The production of dental care in the British National Health Service. Paper presented at the World Congress on Health Economics, Leiden, 1980.

19. Lave C. A. and March J. G. An Introduction to Models in the Social Sciences. Harper \& Row, New York, 1975.

20. Van Groenestijn M. A. J., Maas-de Waal C. J., Mile- 
man P. A. and Swallow J. N. The ideal dentist. Soc. Sci. Med. 14A, 533-540. 1980.

21. There is another kind of costs, the so-called opportunity costs, i.e. the opportunities foregone by choosing a certain alternative. It is difficult to make assumptions on these costs. The opportunity costs include foregone earnings, waiting time, duration of treatment, travel time etc. Foregone earnings are only relevant for the self-employed. some of whom may be publicly insured; waiting time is possibly less for privately insured patients because of an appointment system; duration of treatment may be longer for privately insured patients; travel time may be shorter for those having a car.

22. This general scheme is applicated by Wippler R. The generation of oligarchic structures in constitutionally democratic organizations. In Theoretical Models and Emperical Analyses (Edited by Raub W.). Explanatory Sociology Publications, Utrecht, 1982.

23. We have used data on the density of dentists for 1969 , because these were available from an earlier analysis of the geographical distribution of dentists; see Ref. [3]. 